Network Working Group

Request For Comments: 1303
K. McCloghrie Hughes LAN Systems

M. Rose

Dover Beach Consulting

February 1992

\title{
A Convention for Describing SNMP-based Agents
}

Status of This Memo

This memo provides information for the Internet community. It does not specify an Internet standard. Distribution of this memo is unlimited.

Abstract

This memo suggests a straight-forward approach towards describing SNMP-based agents.

Table of Contents

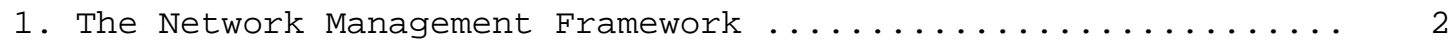

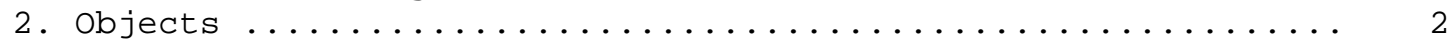

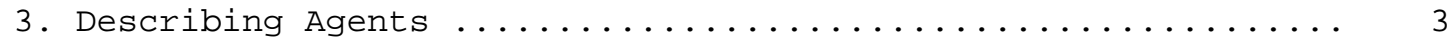

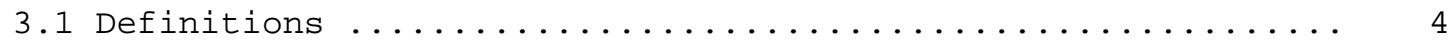

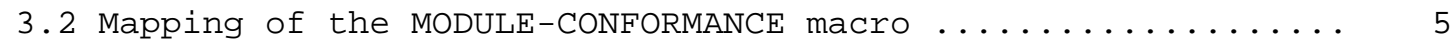

3.2 .1 Mapping of the LAST-UPDATED clause ................ 6

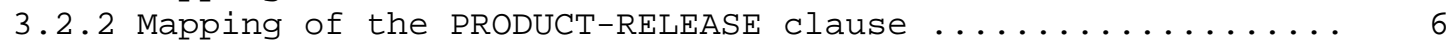

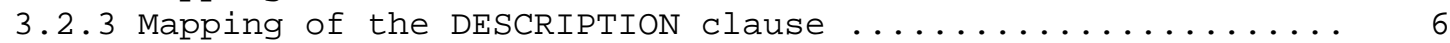

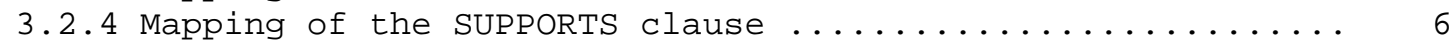

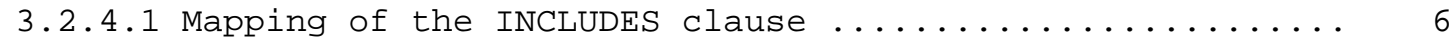

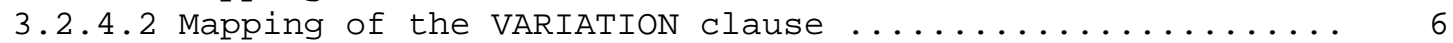

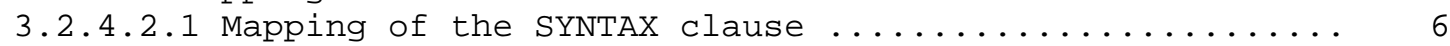

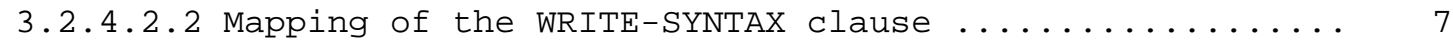

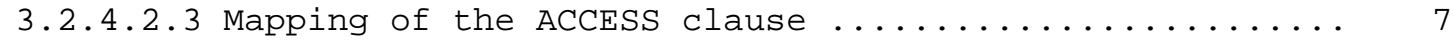

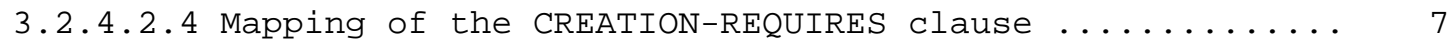

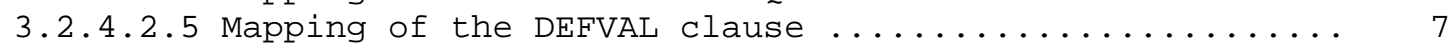

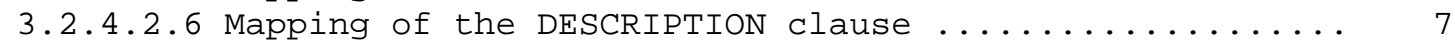

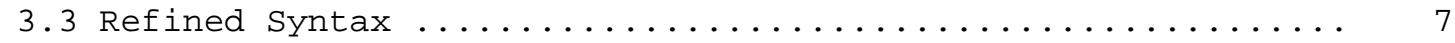

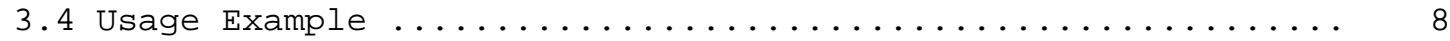

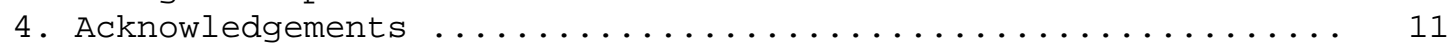

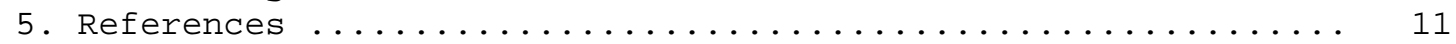

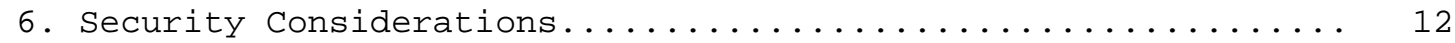

7. Authors' Addresses............................. 12 
1. The Network Management Framework

The Internet-standard Network Management Framework consists of three components. They are:

RFC 1155 [1] which defines the SMI, the mechanisms used for describing and naming objects for the purpose of management. RFC 1212 [2] defines a more concise description mechanism, which is wholly consistent with the SMI.

RFC 1213 [3] which defines MIB-II, the core set of managed objects for the Internet suite of protocols.

RFC 1157 [4] which defines the SNMP, the protocol used for network access to managed objects.

The Framework permits new objects to be defined for the purpose of experimentation and evaluation.

2. Objects

Managed objects are accessed via a virtual information store, termed the Management Information Base or MIB. Within a given MIB module, objects are defined using RFC 1212's OBJECT-TYPE macro. At a minimum, each object has a name, a syntax, an access-level, and an implementation-status.

The name is an object identifier, an administratively assigned name, which specifies an object type. The object type together with an object instance serves to uniquely identify a specific instantiation of the object. For human convenience, we often use a textual string, termed the OBJECT DESCRIPTOR, to also refer to the object type.

The syntax of an object type defines the abstract data structure corresponding to that object type. The ASN.1[5] language is used for this purpose. However, RFC 1155 purposely restricts the ASN.1 constructs which may be used. These restrictions are explicitly made for simplicity.

The access-level of an object type defines whether it makes "protocol sense" to read and/or write the value of an instance of the object type. (This access-level is independent of any administrative authorization policy.)

The implementation-status of an object type indicates whether the object is mandatory, optional, obsolete, or deprecated. 


\section{Describing Agents}

When a MIB module is written, it is divided into units of conformance termed groups. If an agent claims conformance to a group, then it must implement each and every object within that group. Of course, for whatever reason, an agent may implement only a subset of the groups within a MIB module. In addition, the definition of some MIB objects leave some aspects of the definition to the discretion of an implementor.

Practical experience has demonstrated a need for concisely describing the capabilities of an agent with regards to the MIB groups that it implements. This memo defines a new macro, MODULE-CONFORMANCE, which allows an agent implementor to describe the precise level of support which an agent claims in regards to a MIB group, and to bind that description to the sysobjectID associated with the agent. In particular, some objects may have restricted or augmented syntax or accesslevels.

If the MODULE-CONFORMANCE invocation is given to a management-station implementor, then that implementor can build management applications which optimize themselves when communicating with a particular agent. For example, the management-station can maintain a database of these invocations. When a management-station interacts with an agent, it retrieves the agent's sysobjectID. Based on this, it consults the database. If an entry is found, then the management application can optimize its behavior accordingly.

This binding to sysobjectId may not always suffice to define all MIB objects to which an agent can provide access. In particular, this situation occurs where the agent dynamically learns of the objects it supports, for example, an agent supporting SMUX peers via the SMUX protocol [6]. In these situations, additional MIB objects beyond sysobjectID must be used to name other invocations of the MODULE-CONFORMANCE macro to augment the description of MIB support provided by the agent. For example, if an agent's sysobjectID indicates that it supports the SMUX MIB, then each instance of smuxPidentity will indicate another MODULE-CONFORMANCE invocation which is dynamically being supported by the agent. 
3.1. Definitions

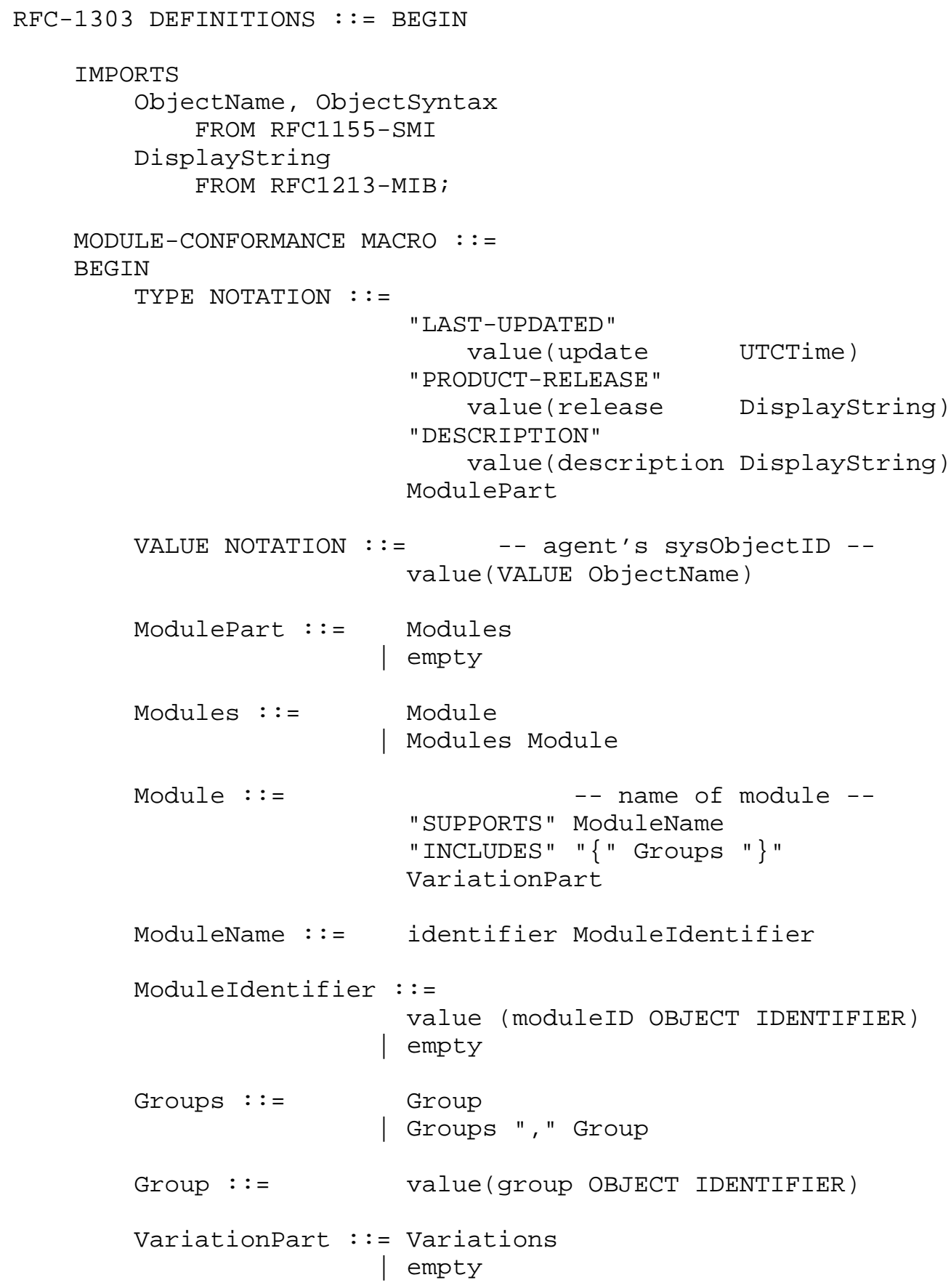




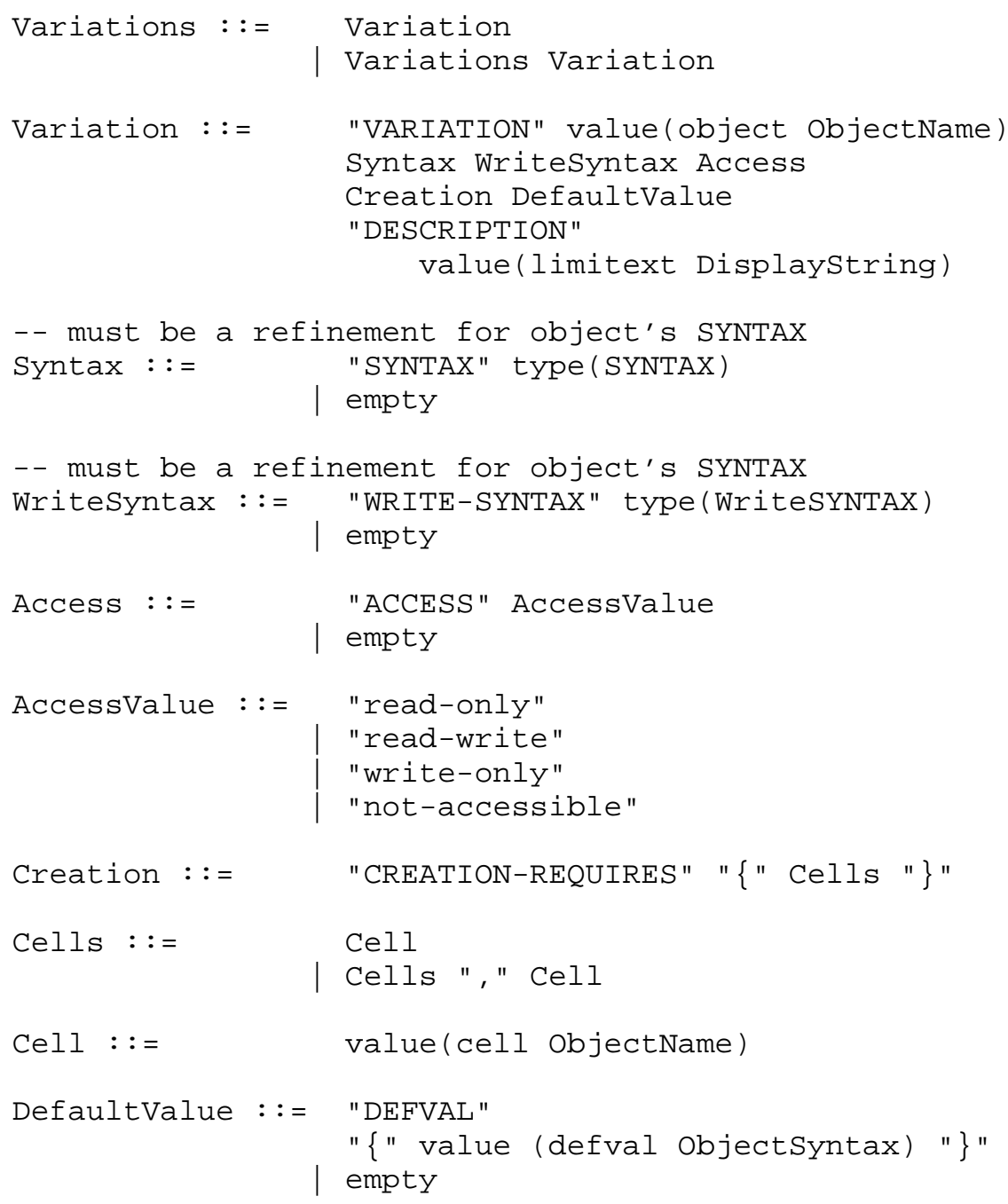




\subsubsection{Mapping of the LAST-UPDATED clause}

The LAST-UPDATED clause, which must be present, contains the date and time that this definition was last edited.

\subsubsection{Mapping of the PRODUCT-RELEASE clause}

The PRODUCT-RELEASE clause, which must be present, contains a textual description of the product release which includes this agent.

\subsubsection{Mapping of the DESCRIPTION clause}

The DESCRIPTION clause, which must be present, contains a textual description of this agent.

\subsubsection{Mapping of the SUPPORTS clause}

The SUPPORTS clause, which need not be present, is repeatedly used to name each MIB module for which the agent claims a complete or partial implementation. Each MIB module is named by its module name, and optionally, by its associated OBJECT IDENTIFIER as well. (Note that only a few MIB modules have had OBJECT IDENTIFIERs assigned to them.)

\subsubsection{Mapping of the INCLUDES clause}

The INCLUDES clause, which must be present for each use of the SUPPORTS clause, is used to name each MIB group associated with the SUPPORT clause, which the agent claims to implement.

\subsubsection{Mapping of the VARIATION clause}

The VARIATION clause, which need not be present, is repeatedly used to name each MIB object which the agent implements in some variant or refined fashion.

\subsection{Mapping of the SYNTAX clause}

The SYNTAX clause, which need not be present, is used to provide a refined SYNTAX for the object named in the correspondent VARIATION clause. Note that if this clause and a WRITE-SYNTAX clause are both present, then this clause only applies when instances of the object named in the correspondent VARIATION clause are read.

Consult section 3.3 for more information on refined syntax. 


\subsection{Mapping of the WRITE-SYNTAX clause}

The WRITE-SYNTAX clause, which need not be present, is used to provide a refined SYNTAX for the object named in the correspondent VARIATION clause when instances of that object are written.

Consult section 3.3 for more information on refined syntax.

\subsection{Mapping of the ACCESS clause}

The ACCESS clause, which need not be present, is used to provide a refined ACCESS for the object named in the correspondent VARIATION clause.

\subsection{Mapping of the CREATION-REQUIRES clause}

The CREATION-REQUIRES clause, which need not be present, is used to name the columnar objects of a conceptual row to which values must be explicitly assigned, by a SNMP set operation, before the agent will create new instances of objects in that row. This clause must not be present unless the object named in the correspondent VARIATION clause is a conceptual row, i.e., has a syntax which resolves to a SEQUENCE containing columnar objects. The objects named in the value of this clause usually will refer to columnar objects in that row. However, objects unrelated to the conceptual row may also be specified.

The absence of this clause for a particular conceptual row indicates that the agent does not support the creation, via SNMP operations, of new object instances in that row.

\subsection{Mapping of the DEFVAL clause}

The DEFVAL clause, which need not be present, is used to provide a refined DEFVAL value for the object named in the correspondent VARIATION clause. The semantics of this value are identical to those of the OBJECT-TYPE macro's DEFVAL clause [2].

\subsection{Mapping of the DESCRIPTION clause}

The DESCRIPTION clause, which must be present for each use of the VARIATION clause, contains a textual description of the variant or refined implementation.

\subsection{Refined Syntax}

The SYNTAX and WRITE-SYNTAX clauses allow an object's syntax to be refined. However, not all refinements of syntax are appropriate. In particular, 
(1) the object's primitive or application type (as defined in the SMI [1]) must not be changed;

(2) an object defined with an SMI type of OBJECT IDENTIFIER, IpAddress, Counter, or TimeTicks cannot be refined; and,

(3) an object defined to have a specific set of values (e.g., an INTEGER with named values) cannot have additional values defined for it.

3.4. Usage Example

Consider how one might document the 4BSD/ISODE "Secure" SNMP agent:

FourBSD-ISODE DEFINITIONS $::=$ BEGIN

IMPORTS

MODULE-CONFORMANCE

FROM RFC-1303

-- everything --

FROM RFCXXXX-MIB

-- everything --

FROM RFC1213-MIB

-- everything --

FROM UNIX-MIB

-- everything --

FROM EVAL-MIB;

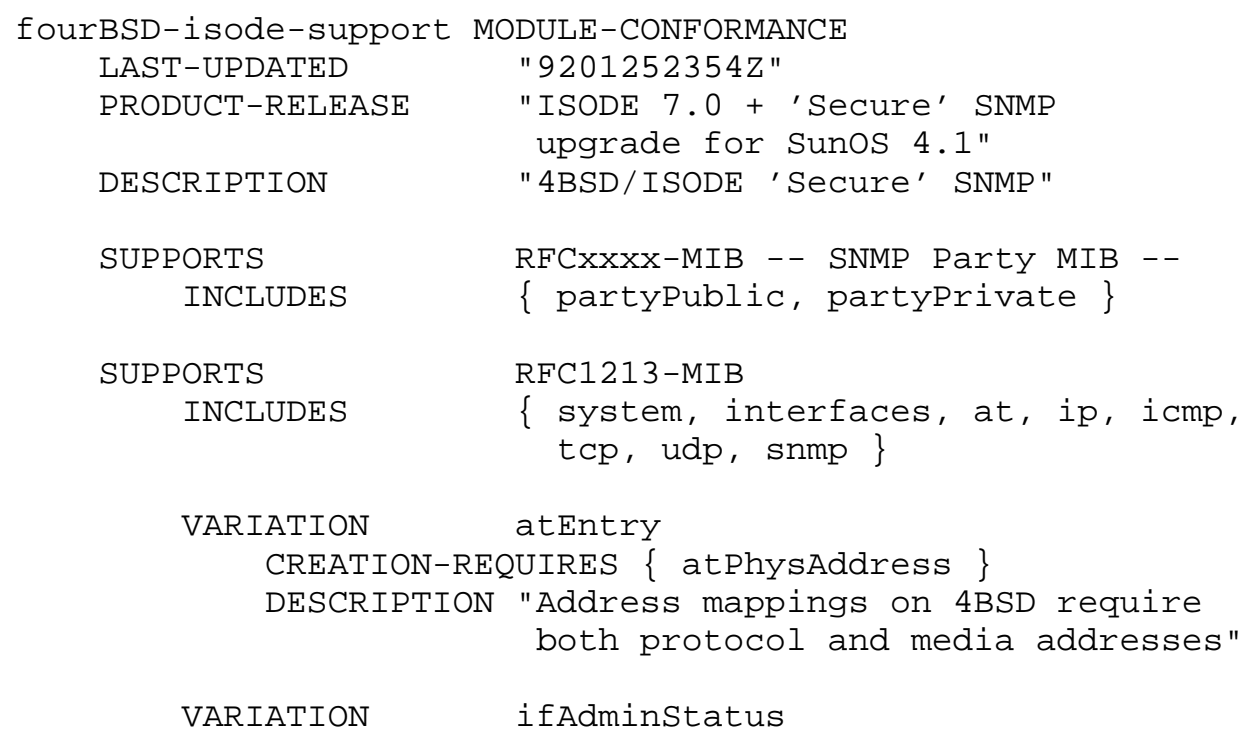




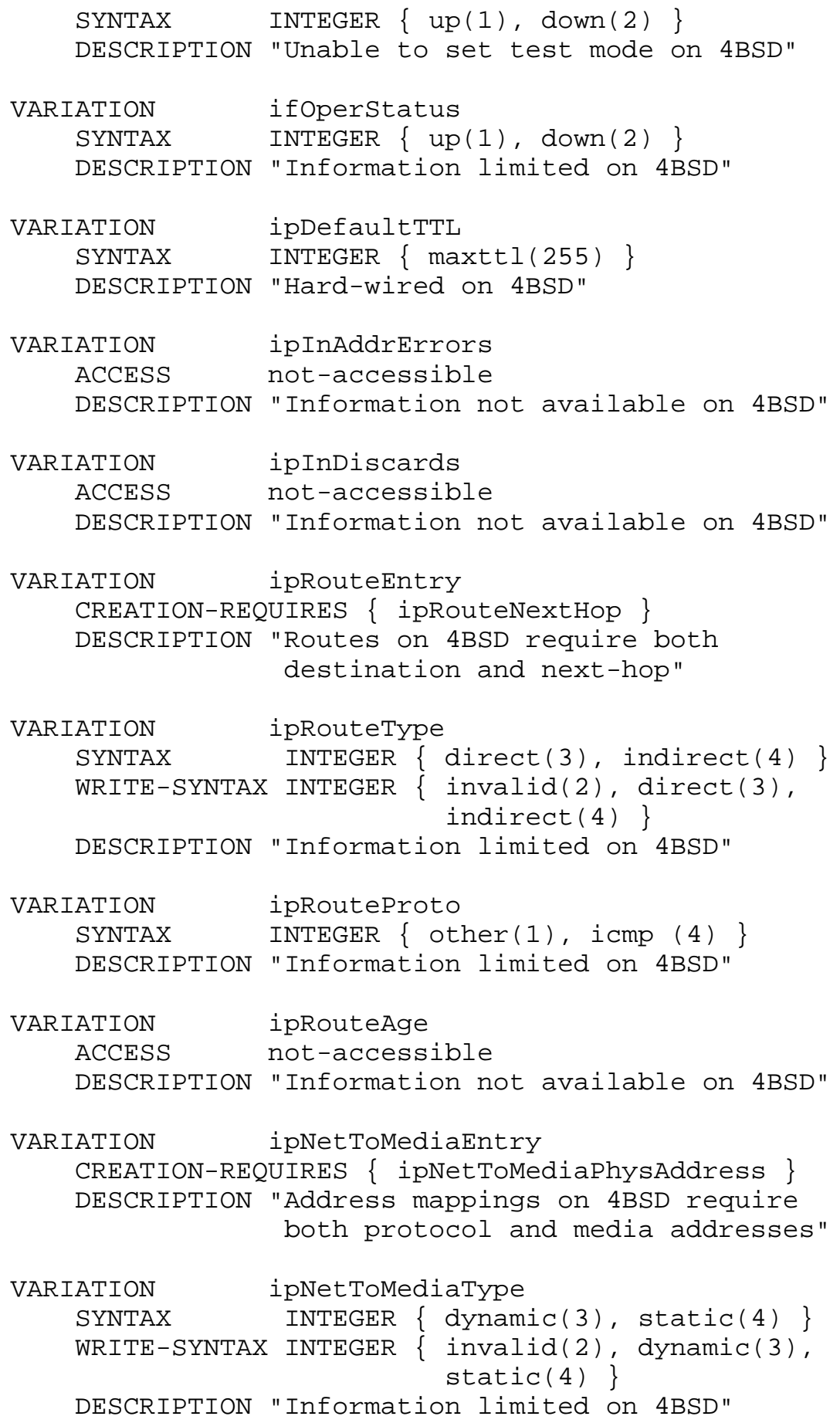




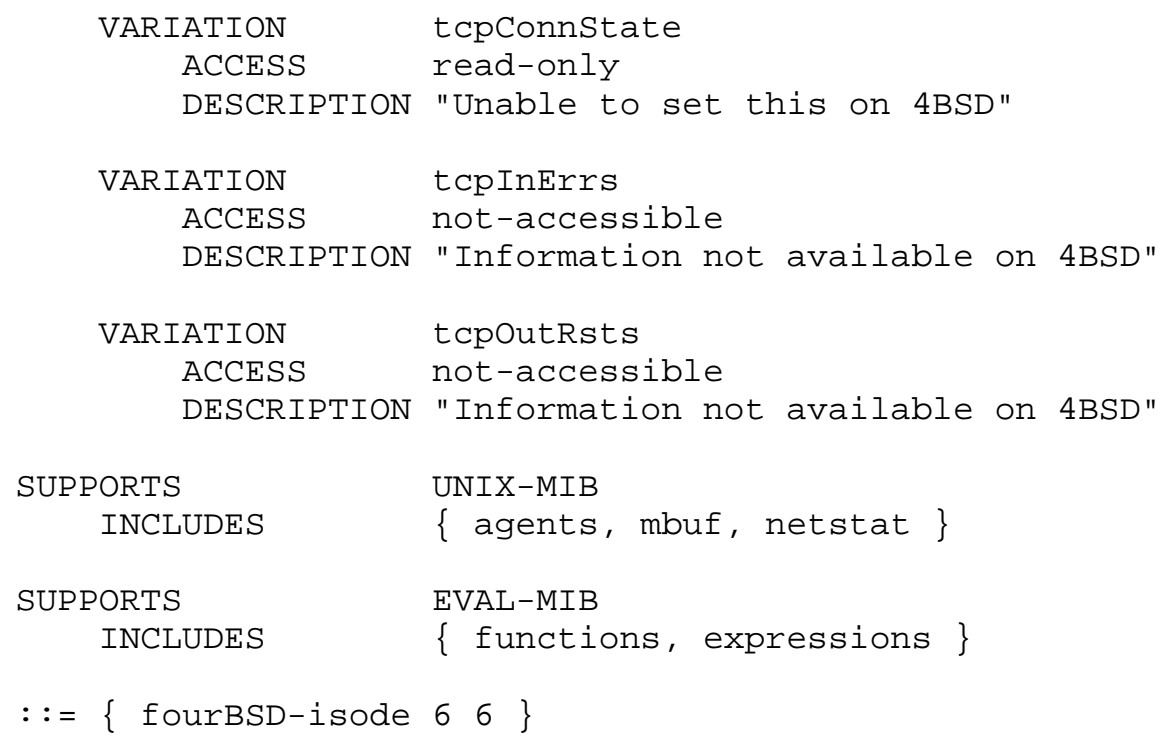


have a restricted syntax, and the object

tcpConnstate

is available only for reading. Note that in the case of the objects

ipRouteType

ipNet ToMediaType

the set of values which may be read is different than the set of values which may be written. Finally, when creating a new row in the atTable, the set-request must create an instance of atPhysAddress. Similarly, when creating a new row in the ipRouteTable, the setrequest must create an instance of ipRouteNextHop. Similarly, when creating a new row in the ipNetToMediaTable, the set-request must create an instance of ipNetToMediaPhysAddress.

From the UNIX-MIB, all the objects contained in the agents, mbuf, and netstat groups are supported, without variation.

From the EVAL-MIB, all the objects contained in the functions and expressions groups are supported, without variation.

4. Acknowledgements

The authors gratefully acknowledge the comments of Mark van der Pol of Hughes LAN Systems, and David T. Perkins of Synoptics Communications.

5. References

[1] Rose M., and K. McCloghrie, "Structure and Identification of Management Information for TCP/IP-based internets", RFC 1155, Performance Systems International, Hughes LAN Systems, May 1990.

[2] Rose, M., and K. McCloghrie, Editors, "Concise MIB Definitions", RFC 1212, Performance Systems International, Hughes LAN Systems, March 1991.

[3] McCloghrie K., and M. Rose, Editors, "Management Information Base forNetwork Management of TCP/IP-based internets", RFC 1213, Performance Systems International, March 1991.

[4] Case, J., Fedor, M., Schoffstall, M., and J. Davin, "Simple Network Management Protocol (SNMP), RFC 1157, SNMP Research, Performance Systems International, Performance Systems International, MIT Laboratory for Computer Science, May 1990. 
[5] Information processing systems - Open Systems Interconnection Specification of Abstract Syntax Notation One (ASN.1), International Organization for Standardization, International Standard 8824, December 1987.

[6] Rose, M., "SNMP MUX Protocol and MIB", RFC 1227, Performance Systems International, May 1991.

6. Security Considerations

Security issues are not discussed in this memo.

7. Authors' Addresses

Keith McCloghrie Hughes LAN Systems 1225 Charleston Road Mountain View, CA 94043

Phone: (415) 966-7934

EMail: kzmahls.com

Marshall T. Rose Dover Beach Consulting, Inc. 420 Whisman Court Mountain View, CA 94043-2112

Phone: (415) 968-1052

EMail: mroseddbc.mtview.ca.us 